\title{
Menstrual Cycle Effects on Performance of Mental Arithmetic Task
}

\author{
Keiko Kasamatsu ${ }^{1)}$, Satoshi Suzuki ${ }^{2)}$, Michiko Anse ${ }^{2)}$, Mariko F. Funada ${ }^{3)}$, \\ Kyoko Idogawa $^{4)}$ and Satoki P. Ninomija ${ }^{2)}$ \\ 1) Department of Ergonomics, Institute of Industrial Ecological Sciences, \\ University of Occupational and Environmental Health, Japan \\ 2) Department of Integrated Information Technology, College of Science and \\ Engineering, Aoyama Gakuin University, Japan \\ 3) Faculty of Business Management, Hakuoh University, Japan \\ 4) Seitoku University, Japan
}

\begin{abstract}
The purpose of this research was to identify the relationship between task performance and menstrual cycle. The difference of performance on menstrual cycle phase was investigated. The task was the mental arithmetic task which involved the non-sequential and higher order cognitive processes. The duration of the experiment was twenty minutes. Two-way analysis of variance by repeated-measures design was used to examine the differences in task performance between phases and temporal variations. Results showed that there was a significant difference in correct input time during temporal variations though there was no significant difference between phases. Moreover, the relationships between phases and intra-individual variations in task performance were examined using coefficient of variance (CV). CVs were plotted in three dimensions to examine the relationships between intra-individual variations and phases. Based on CVs, the subjects who showed differences were classified into two groups: those with a small difference in three phases and those with a difference every phase. The phase which indicated large CV changed with individuals. J Physiol Anthropol 21 (6): 285-290, 2002 http://www.jstage.jst.go.jp/en/
\end{abstract}

Keywords: menstrual cycle, task performance, female, mental task, VDT task

\section{Introduction}

In Japan, the participation of women in the labor force is considered important, and the number of female workers has increased in many fields. Moreover, in Japan, an equal employment opportunity law as well as basic laws for a gender-equal society has been enacted. Working conditions for women have been examined and improved. Nevertheless, the physical and mental stress involved in jobs and in daily life may have negative effects, and there are still many problems related to female health that remain to be discussed. The menstrual cycle is one of these remaining issues.

The biological rhythm of women is typically that of the menstrual cycle. This biological rhythm is influenced by diverse factors, which can have various side effects. The normal menstrual cycle can easily become erratic in response to extreme stress, and some women may suffer from Premenstrual Syndrome (PMS) and amenorrhea (Woods et al., 1998).

Several previous reports have found a close relationship between the biological rhythm of the menstrual cycle and task performance. Walker (1997) examined previous theories and studies which had considered the relationships between menstrual cycle and psychological state. Walker also examined the relationship between cognition and task performance and states that women's beliefs about and attitudes towards menstruation are diverse, and that the relationship between the menstrual cycle and cognitive processes is similarly variable. Asso (1987) examined sex differences on circadian variations in performance, and the relationship between performance and menstrual and circadian rhythms. Dalton and Holton (1999) reported that certain mental and physical capacities change in women in relation to the menstrual cycle.

Moos (1968) considered the effects of the menstrual cycle in view of subjective data and created the Menstrual Distress Questionnaire (MDQ) to facilitate accurate evaluation of a woman's psychological status during each 
menstrual phase. The MDQ measures responses regarding the physical and psychological symptoms experienced during the menstrual cycle. The results reveal that women primarily report having symptoms in the premenstrual and intermenstrual phases. Moos (1968) also classified mental symptoms attributed to the menstrual cycle, and in a later work (Moos et al., 1977) reports on an attempt to develop an empirical typology of menstrual cycle symptoms.

Hartley et al. (1987) described a specific relationship between memory and the menstrual cycle, in which, during ovulation, linguistic inference was slower in complex sentences and scores for recalling thesaurus lists were lower; furthermore, during premenstruation, women showed decreased recollection of aurally presented thesaurus lists. Additionally, the subjects reported pain during premenstruation on the MDQ. Kumari and Corr (1996) suggested that the effects of the menstrual cycle, conceptualized as partly arousal-based, interact with situational manipulations of arousal to influence performance on intelligence tests.

In contrast, Birch et al. (1999) reported only negligible effects of the eumenorrheic menstrual cycle on both manual lifting capacity and the perception of lifting ability. Furthermore, the subjective response to lifting, in the form of perception of exertion and experience of acute back pain, was unaffected by specific menstrual cycle phases. Nakatani et al. (1993) reported that the menstrual cycle did not influence cognitive, physiological, or subjective performance during digit identification. Gamberale (1985) reviewed the experimental results of the effects of menstruation on task performance and subsequently produced guidelines offering recommendations for suitable working conditions for women. This report indicates that the desire to perform to the best of one's ability was most affected by motivation and perceived exertion.

Nevertheless, it is a fact that some women experience physical and emotional symptoms related to the menstrual cycle, and it is believed that the menstrual cycle affects some women's capacity to work. While responses to menstrual phase will vary from person to person, a large sample size may offset those differences and produce a consistent phase-related pattern. Therefore, it is necessary that the effect of the menstrual cycle on different individuals be examined and compared. A brief overview of the related literature illustrates the diversity of results obtained from studies investigating the effects of the menstrual cycle on various types of work and on specific physiological tasks. However, a few studies to date have investigated the relationship between the menstrual cycle and task performance.

In this study, we examined the effects of the menstrual cycle on task performance, using a mental arithmetic task considered to involve non-sequential and higher order cognitive processes. It is difficult to quantify the exact degree of influence of job stress. We selected subjects whose jobs were not stressful, to suppress the influence of job stress on our findings.

\section{Methods}

\section{Menstrual cycle}

The menstrual cycle is generally classified into four phases: menstruation, the follicular phase, ovulation, and the luteal phase. These are defined as follows: menstruation, from the first date of bleeding until five days after the first date of bleeding; follicular phase, between menstruation and ovulation; ovulation, the day when basal body temperature is at its lowest; and luteal phase, between ovulation and menstruation.

We selected menstruation, the follicular phase, and the luteal phase as indices of physical changes. These phases are considered important due to known remarkable changes associated with them (Anse et al., 1994; Kasamatsu et al., 1997, 1999).

\section{Subjects}

The inclusion criteria for subjects in this study were as follows: single women with a regular menstrual cycle, who did not take hormones or other drugs, were not pregnant, and who did not smoke during the six months prior to the experiment. To define the menstrual cycle, the basal body temperature was taken and recorded every morning for three months prior to the beginning of the experiment. The basal body temperature of all subjects was divided into low and high temperature periods, the number of days in the menstrual cycle was determined to be within the normal range, and the subjects' menstrual cycle was confirmed to be fairly regular. All subjects were college students, of 20-23 years of age. A total of 18 subjects (designated A-R) performed the mental arithmetic task during each of the three phases.

\section{Experimental procedure for the mental arithmetic task}

This experiment involved a mental arithmetic task involving the addition of two digits (Fig. 1). The addition problem was shown on a video display terminal (VDT), and there is no answer that the same figure queues up such as 22,33 . The screen did not advance to the next problem until a correct answer was entered using the number pad. If the input answer was correct, the next problem was displayed for 0.5 seconds, and the process was repeated. If the answer was incorrect, the system waited for the correct answer to be entered. The physiological measurement indices were ECG (electrocardiogram) and EEG (electroencephalogram). The analytical results of these indices will be reported in another paper.

The duration of the experiment was twenty minutes. 


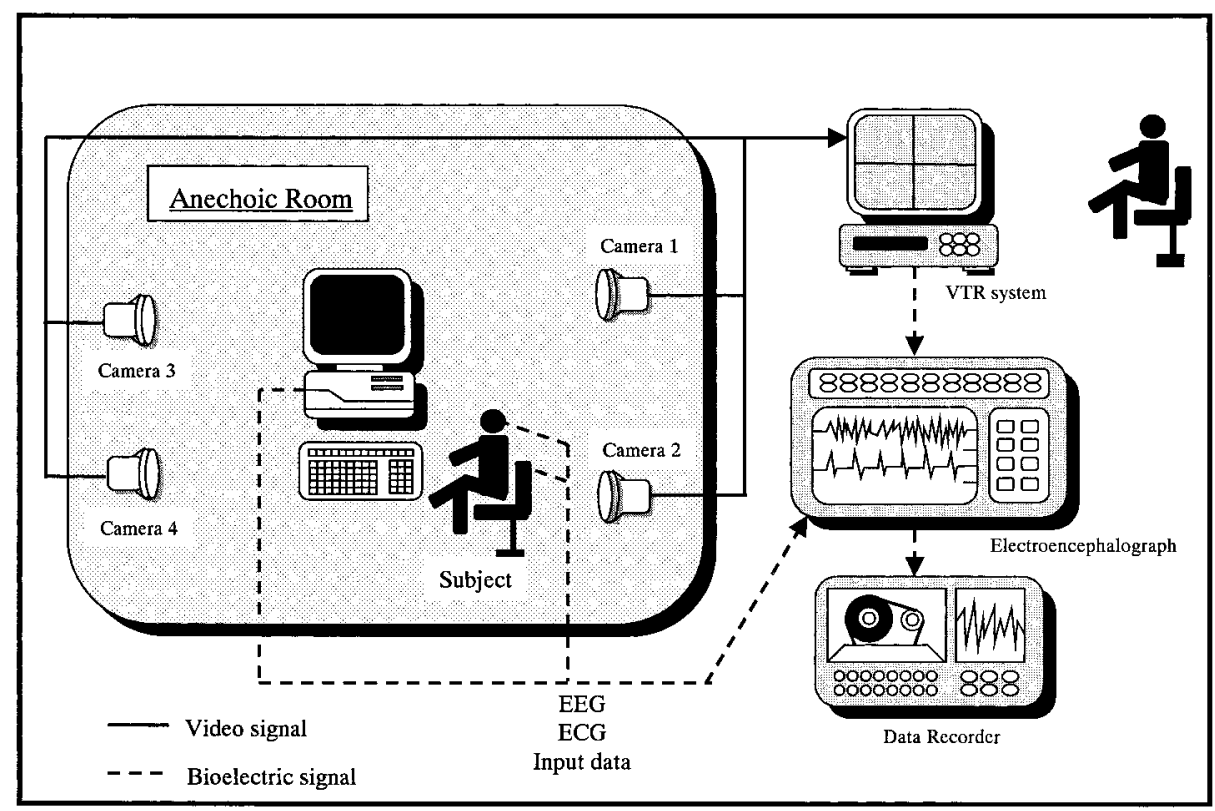

Fig. 1 Mental arithmetic task system chart

Subjects inputted the answers of a couple of two digits addition problems shown on the VDT display in the anechoic room. Subjects were observed by CCD camera. The correct input time, the number of answers and error rate as task performance indices and EEG as physiological indices were measured and recorded.

The correct input time was measured as the task performance index. The correct input time was defined as the interval between when the figure was first displayed to the moment at which the correct answer was entered.

\section{Analytical methods}

Variations trends in the correct input time for each menstrual phase were examined. Two-way analysis of variance by repeated-measures design with the factors being menstrual phases and time course was performed in order to examine the differences between phases and between the influences of time course. Moreover, the interaction between menstrual phases and time course and multiple comparison of these factors were examined.

Individuals experience the effects of the menstrual cycle differently, though there are the large or small differences in its effect. When considering the influence of the menstrual phase on the woman as a whole, the influence between the phases may offset by an individual difference. Therefore, it is necessary that the effect of the menstrual cycle on different individuals be examined and compared. Thus, the coefficient of variation (CV) value in every subject was plotted in a three-dimensional graph and was calculated in order to investigate the relationships between phases and the intra-individual variations of correct input time. However, it is suggested that in the future each group with similar tendencies should be classified together and be examined in terms of the phase effect group-by-group.

\section{Results}

Fig. 2 shows the trend of temporal variations on the correct input time for the 18 subjects. There was not a large difference among three phases, however there was a tendency that the variation in the follicular phase was small. Average value and standard deviation of the follicular phase were the minimum of all of three phases (Fig. 3).

Two-way analysis of variance by repeated-measures design with the factors of phases and temporal variations was performed in order to examine the differences between the three menstrual phases and between the influences of temporal variations on the task performance. The result of the analysis of variance indicated a significant difference between temporal variations $(\mathrm{F}(19,323)=3.67, \mathrm{p}<0.01)$. There was no significant difference in the main effect of the phases, and in the interaction between phase and temporal variation. Then, a multiple comparison using least significant difference (LSD) was examined on account of there being a significant difference between temporal variations. The results indicated a significant difference between the first half (experimental time 0-8) and second half (experimental time 11-20) of the experiment.

In addition, CVs were plotted in three dimensions, as shown in Fig. 4, in order to elucidate the relationships between intra-individual variations and phases. Based on this figure, the subjects who showed differences were classified into two groups: those with a difference (the 


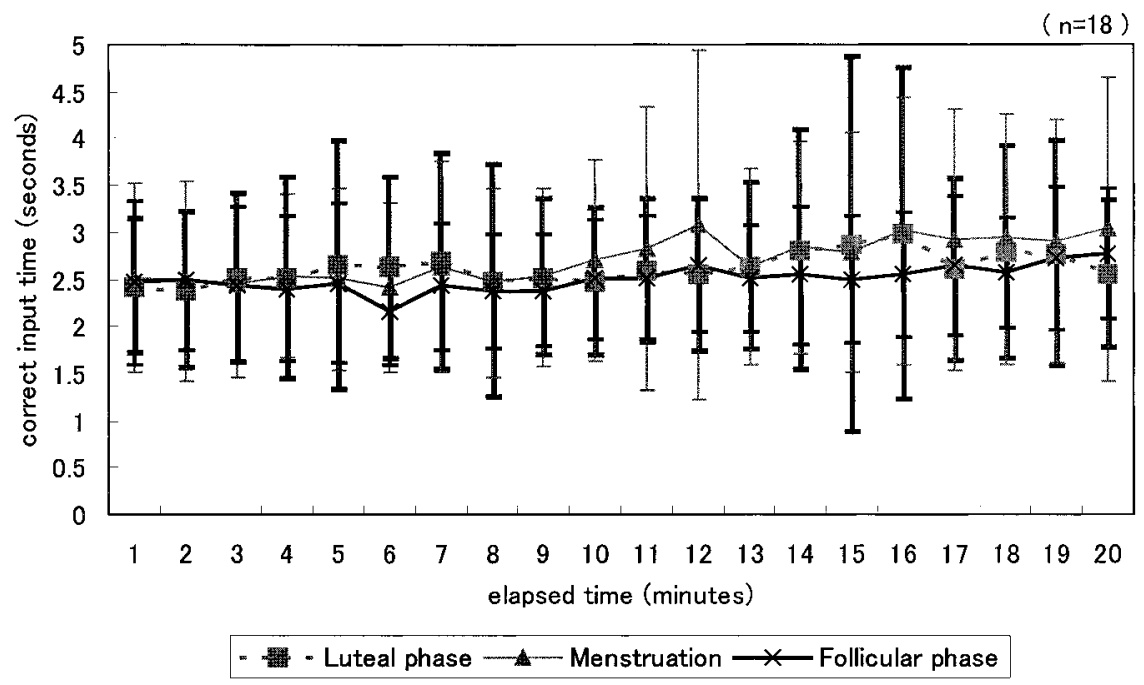

Fig. 2 Temporal variations on the correct input time

This figure shows the trend of temporal variation on correct input time as for 18 subjects. This graph has mean and \pm standard deviation.

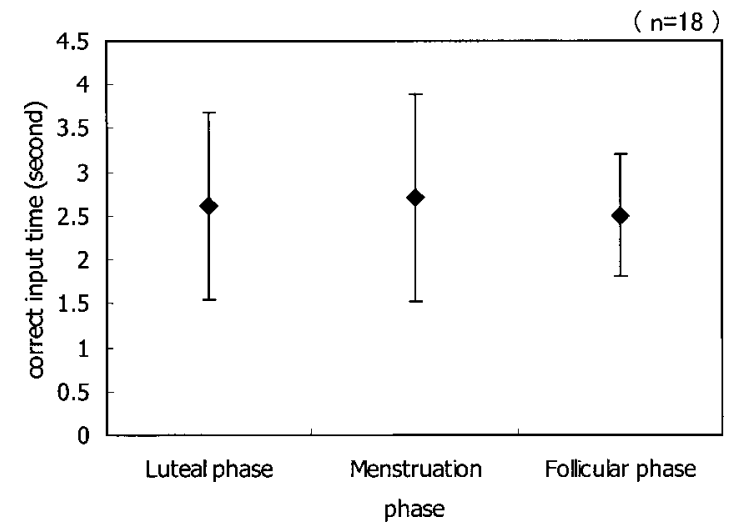

Fig. 3 The average value and standard deviation in three phases This figure shows the average value and the standard deviation on every phase. The average value and the standard deviation in follicular phase are the smallest of three phases.

subject A-D, J-N, R) every phase and those with a small difference (the subject $\mathrm{E}-\mathrm{I}, \mathrm{O}-\mathrm{Q}$ ) in three phases. The phase which indicated large CV changed with individuals. The subjects whose CV values were at their maximum during menstruation are located in the area to the left, diagonally, from the subjects whose CV values were at their maximum during the luteal phase, and those whose $\mathrm{CV}$ values were at their maximum during the follicular phase are located between them.

\section{Discussion}

According to the results of two-way analysis of variance by repeated-measures design, there was a significant difference in correct input time during temporal variations, on the other hand, there were no significant differences between phases and in the interaction between phase and temporal variation. Therefore, there was no difference on temporal variations between phases. However, the average value and standard deviation were the minimum in the follicular phase. As a result of LSD on temporal variations, the task performance on the second half (experimental time 11-20) was lower than the first half (experimental time 0-8).

Based on the CV of correct input time, there are some types of variation in the individual that are caused by variation between phases. In some cases, such as the subjects E-I and O-Q, the CV was low in all three phases and there was no variation between phases regarding the task performance. On the other hand, some subjects showed variation in the $\mathrm{CV}$ of each phase. The task performance was not stable, suggesting that some subjects were influenced by physical and mental symptoms that were induced by phases of the menstrual cycle. The subjects whose CV was at its maximum value during menstruation were located close to the area of the menstruation/follicular phase on the planar graph, while those whose $\mathrm{CV}$ was at its maximum value during the luteal phase were located close to the area of the luteal phase/follicular phase on the planar graph. Thus, the reason for the variation in CV's distribution by phases was considered to be the following. There was a difference in the degree of influence on the task with regard to complaints concerning the menstrual cycle in each phase for every subject. The individual variation factor was found to be strongly related to the effect of the phase of the menstrual cycle in the performance of the mental arithmetic task, specifically, in correct input time.

Nakatani et al. (1993) found that performance on cognitive and physiological tasks was not affected by 


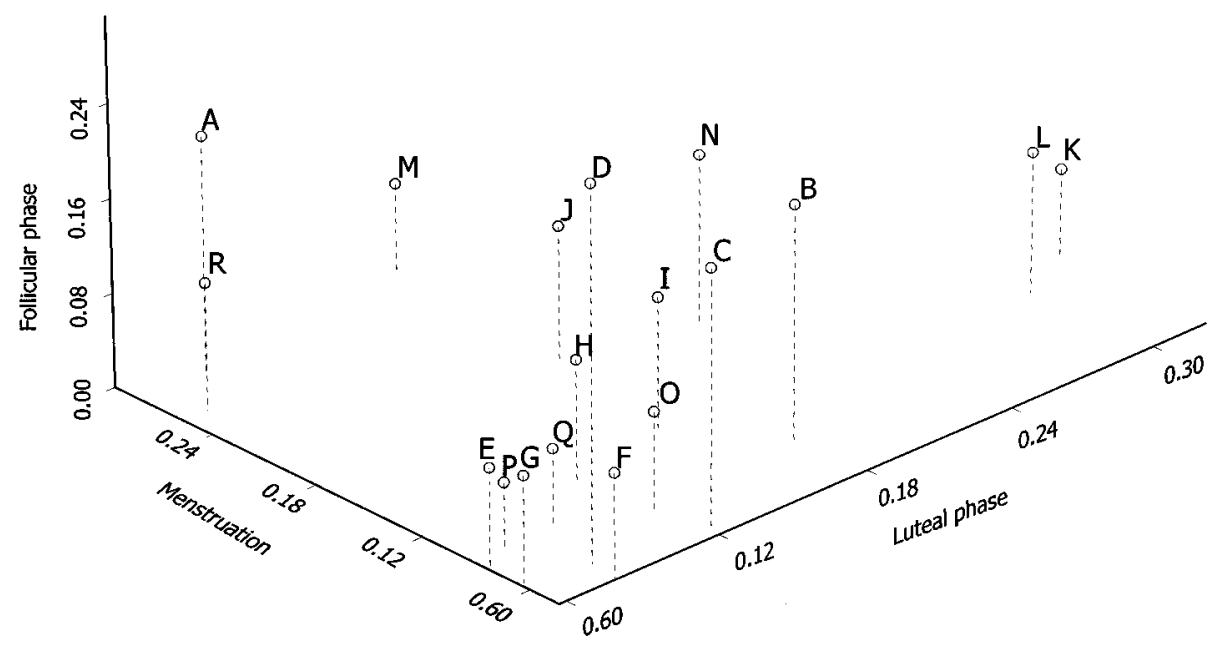

Fig. 4 The positions of coefficient variance in three phases

Coefficient variance was plotted in three dimensions to examine the relationships between intra-individual variation and phases. From this figure, the subjects (A-D, J-N, R) with a large difference and the subjects (E-I, O-Q) with a small difference were classified into subjects.

menstrual cycle. Birch et al. (1999) also reported no differences in manual lifting performance. Similarly, our study did not find differences between task performance during the different menstrual phases. Further research is needed to investigate whether there was no significant difference because of this experimental task, or there was no significant difference originally on the task performance on the phases of the menstrual cycle. Nevertheless, there were the variations between individuals in every phase. Accordingly, the influence of the menstruation cycle should be taken into consideration regarding the performance of mental tasks. We speculate that the menstrual cycle may have a different effect on the performance of different types of tasks.

Many women occasionally use oral contraceptives for relief from menstrual distress. Although oral contraceptives have been available for many years in other countries, they were not easily obtainable in Japan until recently in June of 1999, and the predicted number of women who take the pill in Japan is expected to rise considerably in the future. In this study, none of the subjects had previously taken the pill. Hudgens et al. (1988) reported that normally cycling women, women on menstrual cycles generated by the regular ingestion of oral contraceptives, and men were compared for task performance, and that the ingestion of oral contraceptives influenced task performance being different from normally cycling women. Thus, we believe that the data provided by our subjects will be useful as a control group for future research purposes.

There were differences in performance even in our subjects, who had little job stress. It is worth mentioning that these experimental results might not parallel those for women having important duties or working in a stressful job. In the future, the relationship between physiological cycle and task performance will need to be clarified to elicit optimum performance during the various phases of the menstrual cycle. It is well known that women are influenced physiologically and psychologically by the menstrual cycle. The results of this study serve to deepen our understanding of the effects of the menstrual cycle in task performance. We believe that our data will be useful in future research on the various effects of the menstrual cycle and its influence in the performance of different types of tasks. This information will aid in the development of safety control guidelines for women in the workplace. In future, the effects of the menstrual cycle will be examined with particular attention to subjective assessment, physiological indices, and task performance.

Acknowledgments The authors wish to acknowledge the volunteer subjects and their superiors for allowing the study to be performed.

\section{References}

Anse M, Kasamatsu K, Funada FM, Idogawa K, Ninomija PS (1994) Effect of menstrual cycle on the stability of the task performance in the monotonous work. Proc of 16th Annual Int Conf of the IEEE-EMBS: 311-312

Asso D (1987) Cyclical variations. In M.A.Baker ed. Sex differences in human performance. Chichester, Wiley, $55-80$

Birch KM, Reilly T (1999) Manual handling performance: the effects of menstrual cycle phase. Ergonomics 42: $1317-1332$

Dalton K, Holton W (1999) Once a month-Understanding and treating PMS-. 6th ed. Hunter House Inc., Alameda, 44-80 and 159-171

Gamberale F (1985) Effects of menstruation on work 
performance. Ergonomics 28: 119-123

Hartley LR, Lyons D, Dumme M (1987) Memory and menstrual cycle. Ergonomics 30: 111-120

Hudgens GA, Fatkin LT, Billingsley PA, Mazurczak J (1988) Hand Steadiness: effects of sex, menstrual phase, oral contraceptives, practice, and handgun weight. Human Factors 30: 51-60

Kasamatsu K, Anse M, Funada FM, Idogawa K, Ninomija PS (1997) Research concerning influence on work efficiency of analog and digital VDT work caused by menstrual cycle. Proc of Fifth Int Sci Conf on WWDU: 47-48

Kasamatsu K, Anse M, Funada FM, Idogawa K, Ninomija PS (1999) Effect of menstrual cycle on monotonous works demand high awaking conditions. Humancomputer interaction: Ergonomics and user interfaces, volume 1 of the Proc of the 8th Int Conf on HumanComputer Interaction: 167-172

Kumari V, Corr PJ (1996) Menstrual cycle, arousalinduction, and intelligence test performance. Psychol Rep 78: 51-58

Moos RH (1968) The development of a menstrual distress questionnaire. Psychosom Med 3: 853-867

Moos RH and Leiderman DB (1977) Toward a menstrual cycle symptom typology. J Psychosom Res 22: 31-40 Nakatani C, Sato N, Matsui M, Matsunami M, Kumashiro M (1993) Menstrual cycle effects on a VDT-based simulation task: cognitive indices and subjective ratings. Ergonomics 36: 331-339

Walker AE (1997) The menstrual cycle. 1st ed. Routledge, London and New York, 59-114

Woods NF, Lentz MJ, Mitchell ES, Heitkemper M, Shaver J, Henker R (1998) Perceived stress, physiologic stress arousal, and premenstrual symptoms: group differences and intra-individual patterns, Res in Nursing \& Health 21: $511-523$

Received: February 13, 2001

Accepted: August 26, 2002

Correspondence and reprint request to: Keiko Kasamatsu Department of Ergonomics, Institute of Industrial Ecological Sciences, University of Occupational and Environmental Health, Japan

1-1, Iseigaoka, Yahatanishi, Kitakyushu, Fukuoka, 807-8555, Japan

TEL: +81-93-691-7458

FAX: +81-93-692-0392

e-mail:kkasa@med.uoeh-u.ac.jp 